Pacific Journal of Mathematics

COMPATIBLE TOPOLOGIES AND CONTINUOUS 


\title{
COMPATIBLE TOPOLOGIES AND CONTINUOUS IRREDUCIBLE REPRESENTATIONS
}

\author{
KeVIN J. Sharpe
}

\begin{abstract}
Suppose there are two locally compact group topologies on a group and that the sets of irreducible unitary representations of the group continuous with respect to each of the topologies coincide. Then the topologies are equal if they are comparable or there is a normal subgroup open and $\sigma$-compact in one of the topological groups. This is a result of Klaus Bichteler's, but the work presented here represents a much shorter method than that used by Bichteler, using little representation theory, but using results involving compatible topologies: Topologies containing in common a Hausdorff topology.
\end{abstract}

Klaus Bichteler in his papers [1] and [2] presented some rather interesting ideas on the relationships between two locally compact group topologies on a group when the irreducible unitary representations continuous with respect to each of the two topologies coincide. I wish to present here an alternative proof of Bichteler's conclusions, a proof which uses only a few elementary results from representation theory and as such is rather more simple and considerably shorter than the original. Some ideas for proofs can be traced to Bichteler's [2] and Rajagopolan's [5]. However, the key to this proof is found in my work in what I have called compatible topologies. This paper forms part of a Ph.D. thesis submitted to La Trobe University in Melbourne, Australia. The research was supervised by Dr. Graham Elton of that University, and to whom I am much indebted.

I mentioned compatible topologies above; if $\mathscr{A}_{1}$ and $\mathscr{A}_{2}$ are two topologies on the one space, we say $\mathscr{A}_{1}$ and $\mathscr{A}_{2}$ are compatible if there is a Hausdorff topology weaker than both $\mathscr{A}_{1}$ and $\mathscr{A}_{2}$. This and the following theorems are taken from my paper [6].

THeOREM 1. Let $G$ be a group on which are defined two compatible group topologies $\mathscr{A}_{1}$ and $\mathscr{A}_{2},\left(G, \mathscr{A}_{1}\right)$ being locally compact and $\left(G, \mathscr{A}_{2}\right)$ locally countably compact. If there is a nonemty $\mathscr{A}_{2}$-open set which is contained in an $\mathscr{A}_{1}$-Lindelöf set, then $\mathscr{A}_{1} \subseteq \mathscr{A}_{2}$. In particular if $\mathscr{A}_{1}$ and $\mathscr{A}_{2}$ are two compatible locally compact group topologies on a group $G$, and $\left(G, \mathscr{A}_{1}\right)$ is $\sigma$-compact, then $\mathscr{A}_{1} \subseteq \mathscr{A}_{2}$.

THeOREM 2. Let $\mathscr{A}_{1}$ and $\mathscr{A}_{2}$ be two compatible group topologies defined on a group $G$, such that $G$ is $\mathscr{A}_{1}$-locally compact and $\mathscr{A}_{2}$ - 
locally countably compact. Suppose there is a subgroup $U$ of $G$ which is contained in an $\mathscr{A}_{1}$-Lindelöf set and is such that if $\hat{\mathscr{A}}_{1}$ and $\hat{\mathscr{A}}_{2}$ are the natural topologies for $G / U$ from $\mathscr{A}_{1}$ and $\mathscr{A}_{2}$ respectively, we have $\hat{\mathscr{A}}_{1} \subseteq \hat{\mathscr{L}}_{2}$. Then $\mathscr{A}_{1} \subseteq \mathscr{A}_{2}$.

We also need the following Baire category theory result, which can be found as (5.28) on page 42 of [3].

THEOREM 3. A locally countably compact regular space is not the union of a countable number of closed sets all having void interior.

Bichteler introduced a good notation which we will adopt: If $\mathscr{A}_{1}$ and $\mathscr{A}_{2}$ are locally compact group topologies for the group $G$, $\left(G, \mathscr{A}_{1}\right) \sim\left(G, \mathscr{A}_{2}\right)$ will mean that the sets of irreducible unitary representations of $G$ continuous with respect to each of $\mathscr{A}_{1}$ and $\mathscr{A}_{2}$ coincide. Theorem 4 is the result of Bichteler's which we will prove in the series of lemmas following.

THEOREM 4. Let $G$ be a group on which are defined two locally compact group topologies $\mathscr{A}_{1}$ and $\mathscr{A}_{2}$, such that $\left(G, \mathscr{A}_{1}\right) \sim\left(G, \mathscr{X}_{2}\right)$; $G$ is not necessarily abelian. Then $\mathscr{A}_{1}=\mathscr{A}_{2}$ if one of the following is true: (i) $\mathscr{L}_{1}$ and $\mathscr{L}_{2}$ are comparable; or (ii) there is a normal subgroup of $G$ open and $\sigma$-compact in one of $\left(G, \mathscr{A}_{1}\right)$ or $\left(G, \mathscr{A}_{2}\right)$.

Lemma 5. Let $\mathscr{A}_{1}$ and $\mathscr{A}_{2}$ be two locally compact group topologies on a group $G$ such that $\left(G, \mathscr{A}_{1}\right) \sim\left(G, \mathscr{A}_{2}\right)$. Then

(i) $\mathscr{A}_{1}$ and $\mathscr{A}_{2}$ are compatible topologies;

(ii) if $N$ is a normal subgroup of $G$ which is $\mathscr{A}_{2}$-closed, then $N$ is also $\mathscr{A}_{1}$-closed and $\left(G / N, \hat{\mathscr{A}_{1}}\right) \sim\left(G / N, \hat{\mathscr{A}_{2}}\right)$;

(iii) if $U$ is an $\mathscr{A}_{2}$-open subgroup of $G$, then $U$ is $\mathscr{A}_{1}$-closed and every continuous unitary irreducible representation of $\left(U, \mathscr{A}_{2}\right)$ is $\mathscr{A}_{1}$-continuous on $U$.

Moreover, if $\mathscr{A}_{1} \subseteq \mathscr{A}_{2}$, then (iii) becomes:

(iii)' if $U$ is a subgroup of $G$ either $\mathscr{A}_{1}-$ or $\mathscr{A}_{2}$-open, then $U$ is $\mathscr{A}_{1}$ - and $\mathscr{A}_{2}$-closed and $\left(U, \mathscr{A}_{1}\right) \sim\left(U, \mathscr{A}_{2}\right)$.

Proof. (i)-(iii) are precisely Lemma 3.2 of [2]. For (iii)' we only need consider $U$ to be $\mathscr{A}_{2}$-open, and because of (iii) we only need show that every continuous unitary irreducible representation of $\left(U, \mathscr{A}_{1}\right)$ is on $U \mathscr{A}_{2}$-continuous. But this is immediate as $\mathscr{A}_{1} \subseteq \mathscr{A}_{2}$.

We note that all through the forthcoming analysis we store in the back of our minds the fact that two topologies are compatible 
if they satisfy the conditions of Lemma 5.

LeMma 6. Let $\mathscr{A}_{1}$ and $\mathscr{A}_{2}$ be two locally compact group topologies on a group $G$ such that $\mathscr{A}_{1} \subseteq \mathscr{A}_{2}$ and $\left(G, \mathscr{A}_{1}\right) \sim\left(G, \mathscr{L}_{2}\right)$. If $\left(G, \mathscr{A}_{1}\right)$ is also compact and $\left(G, \mathscr{A}_{2}\right)$ is totally disconnected, then $\mathscr{A}_{1}=\mathscr{A}_{2}$ and in particular $\left(G, \mathscr{A}_{2}\right)$ is also compact.

Proof. We can choose a basis at the identity of $\left(G, \mathscr{A}_{2}\right)$ of open compact subgroups. We shall show these subgroups to be also open in $(G, \mathscr{A})$.

Let $K$ be an open compact subgroup of $\left(G, \mathscr{L}_{2}\right)$. If $K=G$, then Theorem 1 gives $\mathscr{A}_{1}=\mathscr{A}_{2}$.

Otherwise suppose $x_{1}$ is in $K^{\prime}$. Then the group generated by $\left\{x_{1}\right\} \cup K$, say $K_{1}$, is $\mathscr{A}_{2}-\sigma$-compact (as it is the countable union of compact sets) and $\mathscr{A}_{2}$-open (as it contains $K$ ). Now $K$ is a proper subgroup of $K_{1}$ and so at least two left translates of it are required to cover $K_{1}$. If $K_{1}=G$ then Theorem 1 gives $\mathscr{A}_{1}=\mathscr{A}_{2}$.

We continue defining $K_{n}$ 's inducively unless we have a $K_{n}=G$, when we cease operations: Suppose $K_{n-1} \neq G$ and let $x_{n} \in K_{n-1}^{\prime}$. Let $K_{n}$ be the group generated by $\left\{x_{n}\right\} \cup K_{n-1} ; K_{n}$ is $\mathscr{A}_{2}-\sigma$-compact and $\mathscr{A}_{2}$-open. At least 2 left translates of $K_{n-1}$ are needed to cover $K_{n}$, and as left translates of a group by different points are disjoint, there will be needed at least $2^{n}$ left translates of $K$ to cover $K_{n}$.

If, for any $n, K_{n}=G$, then $\mathscr{A}_{1}=\mathscr{A}_{2}$. Otherwise, let $L$ be the subgroup $\bigcup_{n=1}^{\infty} K_{n}$, an open $\sigma$-compact subgroup of $\left(G, \mathscr{A}_{2}\right)$. If $L=G$ then $\mathscr{A}_{1}=\mathscr{A}_{2}$; suppose $L \neq G$. We will need at least a countably infinite number of left translates of $K$ to cover $L$; we will show that if $L \neq K$ we need only a finite number, and hence $\mathscr{A}_{1}=\mathscr{A}_{2}$.

Now $\left(L / K, \hat{\mathscr{A}}_{2}\right)$, the quotient space with the natural topology derived from $\mathscr{A}_{2}$, is $\sigma$-compact and discrete, and hence must be countable. Also $L$ and $K$ are closed subgroups of $\left(G, \mathscr{A}_{1}\right)$ from Lemma 5, and therefore $\left.(L / K, \hat{\mathscr{A}})_{1}\right)$ is a compact regular space (see [3] page 38, (5.21) and (5.22)). Theorem 3 again leaps to the rescue and makes $(L / K, \hat{\mathscr{A}})$ a discrete space and $K$ an open subgroup of $\left(L, \mathscr{L}_{1}\right)$. But $\left(L, \mathscr{X _ { 1 }}\right)$ is a compact group and hence only a finite number of left translates of $K$ are needed to cover it.

LeMma 7. Let $\mathscr{A}_{1}$ and $\mathscr{A}_{2}$ be two locally compact group topologies on a group $G$, such that $\mathscr{A}_{1} \subseteq \mathscr{A}_{2}$ and $\left(G, \mathscr{A}_{1}\right) \sim\left(G, \mathscr{A}_{2}\right)$. If $\left(G, \mathscr{A}_{1}\right)$ is totally disconnected then $\mathscr{A}_{1}=\mathscr{A}_{2}$.

Proof. Let $S$ be any compact open subgroup of $\left(G, \mathscr{A}_{1}\right)$. Now 
$\mathscr{A}_{1} \subseteq \mathscr{A}_{2}$ causes $\left(G, \mathscr{A}_{2}\right)$ to be totally disconnected and $S$ to be an $\mathscr{A}_{2}$-open totally disconnected locally compact subgroup of $\left(G, \mathscr{A}_{2}\right)$. Lemma 5 (iii)' also gives $\left(S, \mathscr{A}_{1}\right) \sim\left(S, \mathscr{A}_{2}\right)$, allowing us to apply Lemma 6 to $S$. Then $S$ is an $\mathscr{A}_{2}$-compact, $\mathscr{A}_{1}$-open subgroup of $G$; Theorem 1 swings into action to give $\mathscr{A}_{2} \subseteq \mathscr{A}_{1}$ and hence $\mathscr{A}_{1}=\mathscr{A}_{2}$.

Lemma 8. Let $\mathscr{A}_{1}$ and $\mathscr{A}_{2}$ be two locally compact group topologies on a group $G$ such that $\mathscr{A}_{1} \subseteq \mathscr{A}_{2}$ and $\left(G, \mathscr{A}_{1}\right) \sim\left(G, \mathscr{A}_{2}\right)$. If $\left(G, \mathscr{A}_{1}\right)$ is a Lie group and $\left(G, \mathscr{A}_{2}\right)$ is totally disconnected then $\mathscr{A}_{1}$ and $\mathscr{A}_{2}$ are both discrete.

Proof. Let $C$ be the component of the identity in $\left(G, \mathscr{A}_{1}\right)$, then all the relevant conditions carry down to $C$ as it is $\mathscr{X}_{1}$-open (see Lemma 5).

Let $U$ be an open compact subgroup of $\left(C, \mathscr{A}_{2}\right)$. Then $U$ is a closed subgroup of $\left(C, \mathscr{A}_{1}\right)$ which is $\sigma$-compact. Hence Theorem 1 gives $\left(U, \mathscr{A}_{1}\right)=\left(U, \mathscr{A}_{2}\right)$ and $\left(U, \mathscr{A}_{1}\right)$ is a totally disconnected Lie group (see [4] page 186), and so is discrete. Then $\left(U, \mathscr{A}_{2}\right)$ is discrete and open in $\left(C, \mathscr{A}_{2}\right)$ and $\left(C, \mathscr{A}_{2}\right)$ is discrete. Any subgroup of $C$ is now $\mathscr{A}_{2}$-open and consequently $\mathscr{A}_{1}$-closed. But the only connected Lie group to have this property is a one element group ([5] in Lemma 4$) ; C=\{e\}$, and $\left(G, \mathscr{A}_{1}\right)$ is discrete.

Lemma 9. Let $\mathscr{A}_{1}$ and $\mathscr{A}_{2}$ be two locally compact group topologies on a group $G$ such that $\mathscr{A}_{1} \subseteq \mathscr{A}_{2}$ and $\left(G, \mathscr{A}_{1}\right) \sim\left(G, \mathscr{A}_{2}\right)$. If $\left(G, \mathscr{A}_{2}\right)$ is totally disconnected then $\mathscr{A}_{1}=\mathscr{A}_{2}$.

Proof. There is an open subgroup $U$ of $\left(G, \mathscr{A}_{1}\right)$ such that, if $C$ is the component of the identity in $\left(G, \mathscr{\mathscr { A } _ { 1 }}\right),\left(U / C, \mathscr{\mathscr { A }}_{1}\right)$ is compact (see [4] page 54, 2.3.1). Again we use [4], this time 4.6 on page 175: Let $A$ be any $\mathscr{A}_{1}$-neighborhood of the identity; $A \cap U$ is an $\mathscr{A}$-neighborhood of the identity in $U$ and let $N$ be the corresponding compact normal subgroup of $\left(U, \mathscr{A}_{1}\right)$ which is in $A$ and is such that $(U / N, \hat{\mathscr{A}})$ is a Lie group.

In fact $\left(U / N, \hat{\mathscr{A}_{1}}\right)$ is a locally compact Lie group, $(U / N, \hat{\mathscr{A}})$ a locally compact totally disconnected group (see [3] page 63, (7.11)), $\hat{\mathscr{A}}_{1} \subseteq \hat{\mathscr{A}_{2}}$, and $\left(U / N, \hat{\mathscr{A}_{1}}\right) \sim\left(U / N, \hat{\mathscr{A}}_{2}\right)$ by Lemma 5 . Applying Lemma 8 we have $\left(U / N, \hat{\mathscr{A}}_{1}\right)$ is discrete. Then $N$ is open in $\left(U, \mathscr{A}_{1}\right)$, also in $\left(G, \mathscr{A}_{1}\right)$, and as every $\mathscr{A}_{1}$-neighborhood of the identity contains such an $\mathscr{A}_{1}$-open subgroup, the identity itself must be the intersection of all $\mathscr{A}_{1}$-open subgroups; $\left(G, \mathscr{A}_{1}\right)$ must be totally disconnected. Applying Lemma 7 we obtain: $\mathscr{A}_{1}=\mathscr{A}_{2}$.

THEOREM 10. Let $\mathscr{A}_{1}$ and $\mathscr{A}_{2}$ be two locally compact group 
topologies on a group $G$ such that $\mathscr{A}_{1} \subseteq \mathscr{A}_{2}$ and $\left(G, \mathscr{A}_{1}\right) \sim\left(G, \mathscr{A}_{2}\right)$. Then $\mathscr{A}_{1}=\mathscr{A}_{2}$.

Proof. The component of the identity in $\left(G, \mathscr{A}_{2}\right)$ is a $\sigma$-compact closed normal subgroup of $\left(G, \mathscr{A}_{2}\right)$. By Theorem 2 we would have $\mathscr{A}_{1}=\mathscr{A}_{2}$ if the two natural topologies on the factor group by this component are equal. Noting that the necessary properties descend to the factor group, the previous lemma provides our solutions.

COROLLARY 11. If every unitary irreducible representation of a locally compact group $(G, \mathscr{A})$ is continuous, then $(G, \mathscr{A})$ is discrete.

Proof. Let $\mathscr{A}_{d}$ be the discrete topology for $G$. Then $\mathscr{A} \subseteq \mathscr{A}_{d}$, and $(G, \mathscr{A}) \sim\left(G, \mathscr{A}_{d}\right)$. The above Theorem 10 then gives $\mathscr{A}=\mathscr{A}_{d}$.

COROLlaRY 12. Let $\mathscr{A}_{1}$ and $\mathscr{A}_{2}$ be two locally compact group topologies on a group $G$ such that $\left(G, \mathscr{A}_{1}\right) \sim\left(G, \mathscr{A}_{2}\right)$. Then if $G$ contains a normal subgroup (say $N)$ which is open and $\sigma$-compact in one of the topologies (say $\mathscr{A}_{1}$ ), we have $\mathscr{A}_{1}=\mathscr{A}_{2}$.

Proof. We need only show $N$ to be $\mathscr{A} \mathscr{A}_{2}$-open, and then Theorem 1 gives $\mathscr{A}_{1} \subseteq \mathscr{A}_{2}$ ready for Theorem 10 to be applied.

The group $\left(G / N, \hat{\mathscr{A}}_{1}\right)$ is discrete, and since $N$ is $\mathscr{A}_{1}$-closed and $(G / N, \hat{\mathscr{A}}) \sim\left(G / N, \hat{\mathscr{Z}_{2}}\right)$ (Lemma 5$)$, the previous Corollary 11 gives that $\left(G / N, \hat{\mathscr{A}_{2}}\right)$ is also discrete, that is $N$ is open in $\left(G, \mathscr{A}_{2}\right)$.

Theorem 10 and Corollary 12 constitute Theorem 4. Corollary 11 is the result in Bichteler's earlier paper [1].

\section{REFERENCES}

1. K. Bichteler, On the existence of noncontinuous irreducible representations of a locally compact group, Inventiones Math., 6 (1968), 159-162.

2. - Locally compact topologies on a group and the corresponding irreducible representations, Pacific J. Math., 31 (1969), 583-593.

3. E. Hewitt and K. A. Ross, Abstract Harmonic Analysis, Vol. I: Structure of Topological Groups, Integration Theory, Group Representations, Die Grundlehren der math. Wissenschaften, Band 115, Academic Press, New York; Springer-Verlag, Berlin, 1963.

4. D. Montgomery and L. Zippin, Topological Transformation Groups, Interscience tracts in Pure and Applied Math. 1, Interscience Publishers, New York, 1955.

5. M. Rajagopolan, Topologies in locally compact groups, Math. Ann., 176 (1968), $169-180$.

6. K. J. Sharpe, Compatible topologies and continuous points in topological groups, to appear.

Received October 16, 1973.

la Trobe University, Melbourne, Australia

Present address: Episcopal Divinity School, Cambridge, Mass. 02138 



\section{PACIFIC JOURNAL OF MATHEMATICS}

EDITORS

RICHARD ARENS (Managing Editor)

University of California

Los Angeles, California 90024

R. A. Beaumont

University of Washington

Seattle, Washington 98105

J. DugundJI

Department of Mathematics

University of Southern California

Los Angeles, California 90007

D. Gilbarg and J. Milgram

Stanford University

Stanford, California 94305

\section{ASSOCIATE EDITORS}

E. F. BECKENBACH

B. H. NeumanN

F. WOLF

K. YOSHIDA

\section{SUPPORTING INSTITUTIONS}

UNIVERSITY OF BRITISH COLUMBIA CALIFORNIA INSTITUTE OF TECHNOLOGY

UNIVERSITY OF CALIFORNIA

MONTANA STATE UNIVERSITY

UNIVERSITY OF NEVADA

NEW MEXICO STATE UNIVERSITY

OREGON STATE UNIVERSITY

UNIVERSITY OF OREGON

OSAKA UNIVERSITY

\author{
UNIVERSITY OF SOUTHERN CALIFORNIA \\ STANFORD UNIVERSITY \\ UNIVERSITY OF TOKYO \\ UNIVERSITY OF UTAH \\ WASHINGTON STATE UNIVERSITY \\ UNIVERSITY OF WASHINGTON \\ $\stackrel{*}{*} \stackrel{*}{*}{ }^{*}{ }^{*}{ }^{2}$ AMERICAN MATHEMATICAL SOCIETY \\ NAVAL WEAPONS CENTER
}




\section{Pacific Journal of Mathematics}

\section{Vol. 52, No. $1 \quad$ January, 1974}

David R. Adams, On the exceptional sets for spaces of potentials ............ 1

Philip Bacon, Axioms for the Čech cohomology of paracompacta ............ 7

Selwyn Ross Caradus, Perturbation theory for generalized Fredholm operators ..... 11

Kuang-Ho Chen, Phragmén-Lindelöf type theorems for a system of nonhomogeneous equations ............................ 17

Frederick Knowles Dashiell, Jr., Isomorphism problems for the Baire classes .......

M. G. Deshpande and V. K. Deshpande, Rings whose proper homomorphic images are right subdirectly irreducible . . . . . . . . . . . . . . . . . . . . . . . . .

Mary Rodriguez Embry, Self adjoint strictly cyclic operator algebras .............

Paul Erdős, On the distribution of numbers of the form $\sigma(n) / n$ and on some related

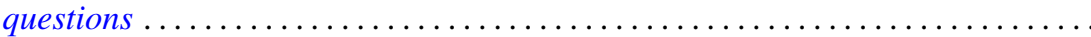

Richard Joseph Fleming and James E. Jamison, Hermitian and adjoint abelian

operators on certain Banach spaces ............................

Stanley P. Gudder and L. Haskins, The center of a poset .................. 85

Richard Howard Herman, Automorphism groups of operator algebras . . . ........

Worthen N. Hunsacker and Somashekhar Amrith Naimpally, Local compactness of families of continuous point-compact relations ....................

Donald Gordon James, On the normal subgroups of integral orthogonal groups ....

Eugene Carlyle Johnsen and Thomas Frederick Storer, Combinatorial structures in

loops. II. Commutative inverse property cyclic neofields of prime-power

order.

Ka-Sing Lau, Extreme operators on Choquet simplexes . . . . . . . . . . . . . . 129

Philip A. Leonard and Kenneth S. Williams, The septic character of 2, 3, 5 and $7 \ldots 143$

Dennis McGavran and Jingyal Pak, On the Nielsen number of a fiber map ........ 149

Stuart Edward Mills, Normed Köthe spaces as intermediate spaces of $L_{1}$ and

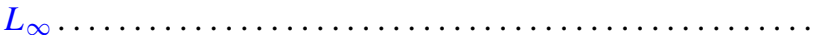

Philip Olin, Free products and elementary equivalence. .

Louis Jackson Ratliff, Jr., Locally quasi-unmixed Noetherian rings and ideals of the principal class.

Seiya Sasao, Homotopy types of spherical fibre spaces over spheres ...

Helga Schirmer, Fixed point sets of polyhedra ...

Kevin James Sharpe, Compatible topologies and continuous irreducible

representations.

Frank Siwiec, On defining a space by a weak base . . . . . . . . . . . . . . . 233

James McLean Sloss, Global reflection for a class of simple closed curves ....... 247

M. V. Subba Rao, On two congruences for primality . .

Raymond D. Terry, Oscillatory properties of a delay differential equation of even

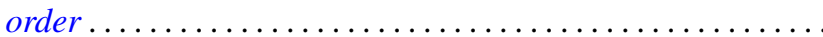

Joseph Dinneen Ward, Chebyshev centers in spaces of continuous functions . .

Robert Breckenridge Warfield, Jr., The uniqueness of elongations of Abelian

groups...

V. M. Warfield, Existence and adjoint theorems for linear stochastic differential

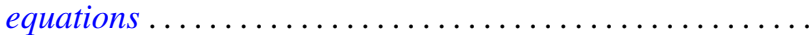

